Original Article

Joumal of Epilepsy Research pISSN 2233-6249 / elSSN 2233-6257

Received June 30, 2019

Revised January 5, 2020

Accepted January 6, 2020

Corresponding author:

Hussein Algahtani, MD

King Abdulaziz Medical City, King Saud

bin Abdulaziz University for Health

Sciences, P.O. Box: 12723, Jeddah 21483 ,

Saudi Arabia

Tel. +966-556633130

E-mail; halgahtani@hotmail.com

\title{
Antiepileptic Drugs Usage in Pregnant Women with Epilepsy in Saudi Arabia
}

\author{
Hussein Algahtani, MD², Bader Shirah, MD², Faisal Alkahtani, MD³ Khalid Alrefaei, MD, \\ Abdulrahman Alamri, MD³ Ahmed Aldarmahi, $\mathrm{PhD}^{3}$ \\ ${ }^{1}$ King Abdulaziz Medical City, King Saud bin Abdulaziz University for Health Sciences, Jeddah; ${ }^{2}$ King Abdullah \\ International Medical Research Center, King Saud bin Abdulaziz University for Health Sciences, Jeddah; ${ }^{3}$ King Saud \\ bin Abdulaziz University for Health Sciences, Jeddah, Saudi Arabia
}

\begin{abstract}
Background and Purpose: Epilepsy is one of the most common neurological disorders requiring continuous treatment during pregnancy. In Saudi Arabia, there is only one publication that studied the outcome of pregnancies in women with epilepsy, published in 1999. The aim of the study is to determine the major congenital malformations in infants resulting from exposure to antiepileptic drugs in pregnant women with epilepsy.
\end{abstract}

Methods: This is a retrospective observational study that was conducted at King Abdulaziz Medical City, Jeddah, Saudi Arabia, involving pregnant women with epilepsy using antiepileptic drugs during pregnancy. We also studied babies born to those mothers. The study period was 5 years from 2014 to 2018 .

Results: Six hundred babies were included in the study, born to 154 mothers with epilepsy using antiepileptic drugs during pregnancy. In addition, there were 111 losses of fetuses before 20 weeks of gestation. The only malformation detected was a ventricular septal defect in one child, whose mother was using polytherapy (valproic acid and levetiracetam). Three babies were born with epilepsy, and four babies had other associated disorders (Down syndrome, osteoporosis, esotropia, and hearing impairment).

Conclusions: The results of this small study are an urgent call for the establishment of congenital malformations registry in Saudi Arabia. In addition, specialized epilepsy clinics utilizing multidisciplinary care are highly recommended. A specific group of interest for such clinics are married women, who have epilepsy and are using antiepileptic drugs since planning of pregnancy is not part of the culture in Saudi Arabia. (2019;9:134-138)

Key words: Epilepsy, Anticonvulsants, Pregnancy, Saudi Arabia

\section{Introduction}

Epilepsy is one of the most common neurological disorders with approximately fifty million affected subjects worldwide. It affects people of all age groups with no sex preference. 'In women, epilepsy usually manifests in the reproductive age group due to changes in hormonal cycles, reproductive alterations, and pregnancy complications. ${ }^{2}$ Antiepileptic drugs are active compounds that target the central nervous system to reduce seizure incidence by different biochemical mechanisms, which eventually reduce pathological hyperexcitability of the cerebral cortex. ${ }^{3}$ In pregnant women with epilepsy, the chances of having babies with congenital malformation increase up to $4-6 \%$ as compared to $2-3 \%$ in the general population. The reason behind this high incidence could be related to exposure to antiepileptic drugs and/or increased risk of maternal seizures. ${ }^{4}$ Important goals in the management of a pregnant woman with epilepsy include controlling seizure episodes, maintaining the mother's wellbeing, and delivering a healthy baby. ${ }^{5}$

Epilepsy is one of the most common neurological disorders requiring continuous treatment during pregnancy. Although women should not take any medication during pregnancy, epilepsy is an exception since status epilepticus or physically dangerous seizures can occur without provocation to many pregnant women with epilepsy. ${ }^{6}$ Neurologists treating pregnant women usually face the dilemma of balancing the risk of developing seizures versus the risk of in-utero drug exposure to the developing fetus. The epileptic mothers should 
participate in decision making, and her concerns and opinions should be addressed. ${ }^{7}$ Prior to planning a pregnancy, neurologists should review current seizure frequency, severity, and used medications. In addition, they should review the history of congenital malformation with previous pregnancies or family history of such malformation. Since many women with epilepsy will continue to take medications during pregnancy, a discussion, which is usually emotional, of the risk of congenital malformation follows. ${ }^{8}$

Many studies have been conducted over the world on pregnant women who are using antiepileptic drugs in their pregnancy. ${ }^{9}$ In Saudi Arabia, there is only one publication, published in 1999, that studied the outcome of pregnancies in women with epilepsy. ${ }^{10}$ The aim of the study is to determine the major congenital malformations in infants resulting from exposure to antiepileptic drugs in pregnant women with epilepsy. This study is also important because of the availability of many new generation antiepileptic drugs, which are known to be safer than the old group.

\section{Methods}

This is a retrospective observational study that was conducted at King Abdulaziz Medical City (KAMC), Jeddah, Saudi Arabia, involving pregnant women with epilepsy using antiepileptic drugs during pregnancy. We also studied babies born to those mothers. The study period was 5 years from 2014 to 2018. The data was collected from KAMC-J, which is one of the tertiary care centers in the western region of Saudi Arabia. All women fulfilling the criteria were included in the study, utilizing the consecutive sampling technique. However, the participants who declined to participate or did not match the study inclusion criteria were excluded. The sample size was the total number of the patient population at the neurology department for the period of the last 5 years. The patients' data were collected from both their electronic and paper-based files from the medical records section in KAMC, complimented by telephone interviews.

All numerical values and demographic data obtained from the questionnaire were calculated and presented as frequency and percentage. Continuous variables were described by using means, median, and standard deviation. All collected data were entered and analyzed by using the IBM Statistical Package for the Social Sciences (SPSS) version 23 (IBM Corp., Armonk, NY, USA). All data collection sheets were anonymous, and confidentiality of information was maintained. This study is conducted under the permission of the Institutional Review Board at King Abdullah International Medical
Research Center (IRB Number: IRBC/1478/17).

\section{Results}

Six hundred babies were included in the study, born to 154 mothers with epilepsy using antiepileptic drugs during pregnancy. Types of epilepsy in those mothers, according to the International League Against Epilepsy 2017 classification, were generalized-onset (81.8\%) and focal-onset (18.2\%). In addition, we identified the cause of all structural epilepsies such as brain tumors and trauma. $40 \%$ of the pregnancies were unplanned. Only six mothers admitted using folic acid during pregnancy. The mean age and weight of mothers were 41 years and $67 \mathrm{~kg}$, respectively. The mean duration of using antiepileptic drugs was 14 years. Monotherapy was used by 135 mothers (87.7\%), while 19 mothers (12.3\%) were using polytherapy (Table 1).

All babies included in the study were exposed to antiepileptic drugs during pregnancy. All babies were born at term (between 38 weeks to 40 weeks), except four babies who were born earlier than 38 weeks. Regarding the type of delivery, 494 births were spontaneous vaginal delivery, while 106 births were done via cesarean section. In addition, there were 111 losses of fetuses before 20 weeks of gestation. The gender distribution showed that 291 were males and 309 were females. The head circumference was in the normal percentile in all of the newborns, and also $98 \%$ of them had normal birth weight. The reference range for normal birth weight was 2.5-4 kg (Table 2).

Table 1. Details of the mothers included in the study

\begin{tabular}{lc}
\hline Variable & Value \\
\hline Number of mothers & $154(100)$ \\
Types of epilepsy in mothers & $126(81.8)$ \\
Generalized-onset & $28(18.2)$ \\
Focal-onset & $6(3.9)$ \\
Using folic acid during pregnancy & $148(96.1)$ \\
Yes & 41 \\
No & 67 \\
The mean age of mothers (years) & 14 \\
The mean weight of mothers (kg) & $19(12.3)$ \\
The mean duration of using antiepileptic drugs (years) & \\
Type of antiepileptic drug therapy & $135(87.7)$ \\
Monotherapy & 19 \\
Polytherapy &
\end{tabular}

Values are presented as number (\%). 
The most commonly used antiepileptic drug during pregnancy was Levetiracetam (396 pregnancies) followed by Lamotrigine (127 pregnancies). The number of pregnancies exposed to Lacosamide was 38 pregnancies. Carbamazepine was used in 51 pregnancies and valproic acid in 20 pregnancies (Table 3).

The only malformation detected was a ventricular septal defect in one child whose mother was using polytherapy (valproic acid and levetiracetam). Three babies were born with epilepsy, who started immediately after birth, and four babies had other associated disorders (Down syndrome, osteoporosis, esotropia, and hearing impairment). In babies born with epilepsy, the cause was high-grade fever in one baby and hypoglycemia in another, but the cause in the third baby was unknown. The type of epilepsy in their mothers was primary generalized epilepsy. Antiepileptic drugs used in these mothers were carbamazepine (one mother), valproic acid (one mother), and combination of carbamazepine and lacosamide (one mother). Regarding the baby born with Down syndrome, the only identified risk factor was mother's increased maternal age (46 years). She was using

Table 2. Details of the babies included in the study

\begin{tabular}{lc}
\hline Variable & Value \\
\hline Type of delivery & \\
Spontaneous vaginal delivery & $494(82.3)$ \\
Cesarean section & $106(17.7)$ \\
Gender of the babies & \\
Male & $291(48.5)$ \\
Female & $309(51.5)$ \\
Childbirth & \\
At term & $596(99.3)$ \\
Preterm & $4(0.7)$ \\
Birth weight & \\
Normal $(2.5-4 \mathrm{~kg})$ & $588(98.0)$ \\
Low (below $2.5 \mathrm{~kg})$ & $12(2.0)$ \\
\hline
\end{tabular}

Values are presented as number (\%).

Table 3. Frequency of babies exposed to antiepileptic drugs during pregnancy

\begin{tabular}{lc}
\hline Drug & Value \\
\hline Levetiracetam & 396 \\
Lamotrigine & 127 \\
Carbamazepine & 51 \\
Lacosamide & 38 \\
Valproic acid & 20 \\
\hline
\end{tabular}

Lamotrigine, which is most likely unrelated to Down syndrome. Diseases or signs affecting babies (osteoporosis, esotropia, and hearing impairment) are most likely unrelated to their maternal epilepsy or antiepileptic drug use.

\section{Discussion}

Careful selection of antiepileptic drugs can lower the potential adverse effects of antiepileptic drugs while maintaining seizure control for the health of not only the mother but also the unborn fetus. ${ }^{11}$ In our study, we detected only one malformation among the babies who were exposed to antiepileptic drugs during pregnancy. This malformation was caused by using polytherapy (valproic acid and levetiracetam).

A systematic review and meta-analysis, published in 2015, including 38 studies from different countries around the world, found that "The usage of antiepileptic drugs is associated with a small but significant increase in adverse pregnancy outcomes such as antepartum and postpartum hemorrhage, spontaneous miscarriage, cesarean section, preterm birth, fetal growth restriction, and congenital malformations". ${ }^{12}$ In our study, the rate of miscarriage was $15.6 \%$ of all pregnancies, which is slightly higher than the reported incidence worldwide (10-15\%). This number could be higher since we did not test routinely for pregnancy before 4 weeks or 4 weeks after the last menstrual period. There was no case of antepartum or postpartum hemorrhage and only one case of intrauterine growth restriction was identified, which was also associated with polytherapy.

Long term teratogenic effects of old generation antiepileptic drugs are well-recognized. However, this effect may not be applied for new generation antiepileptic drugs based on the available data and publications. Significant risk factors, which could cause teratogenicity and are mostly overlooked, include a family history of birth defects, smoking, alcohol intake, and drug abuse. Further studies are needed to identify and recognize the short and long term effects of the new generation antiepileptic drugs. The information on the teratogenic effects of new generation antiepileptic drugs is limited to case reports and pregnancy registries. ${ }^{13}$ In our study, when new generation antiepileptic drugs were used as monotherapy for women with epilepsy, no teratogenic effect was observed. The same is applied for old generation antiepileptic drugs when used alone. However, the sample size for old generation antiepileptic drugs was very small, which indicated a trend in prescribing new generation antiepileptic drugs for pregnant women with epilepsy. 
Monotherapy is safer and has a less teratogenic effect on babies than polytherapy. Among antiepileptic drugs used as monotherapy, intrauterine first-trimester exposure to valproic acid has a higher risk of major congenital malformations compared to other antiepileptic drugs. ${ }^{14}$ In addition, there is a probable relationship between the dose of the antiepileptic drug used and the risk of developing major congenital malformations in the newborn of women with epilepsy. This is particularly true when using valproic acid and lamotrigine. ${ }^{15}$ Additionally, specific types of major congenital malformations are associated with the specific use of an antiepileptic drug. For example, phenytoin and valproic acid contribute to neural tube defects, facial clefts, and hypospadias. ${ }^{16}$ Monotherapy is preferred to polytherapy during pregnancy, and such a target should be achieved in the preconception period. This is specifically true when valproic acid is part of the polytherapy regimen. ${ }^{17}$

According to the Royal College of Obstetricians and Gynecologists in the United Kingdom, $1 \mathrm{mg}$ of intramuscular vitamin $\mathrm{K}$ should be offered to all babies born to women with epilepsy taking enzyme-inducing antiepileptic drugs. This is mainly to prevent hemorrhagic disease of the newborn. The evidence is insufficient to recommend oral administration of vitamin $\mathrm{K}$ to mothers with epilepsy. ${ }^{18}$ In our study, vitamin $\mathrm{K}$ was not used or offered to mothers or newborns. No hemorrhagic disease of the newborn was observed, which could be the reason behind this practice.

All pregnant women with epilepsy should be advised to take daily folic acid $(5 \mathrm{mg})$ prior to conception and until at least the end of the first trimester. ${ }^{19}$ In our study, only six patients admitted using folic acid during pregnancy. We doubt this result since it is a routine practice in our hospital to prescribe folic acid to all pregnant women with or without epilepsy, especially in the first trimester of pregnancy. Another study should be conducted to evaluate the use of folic acid in pregnant women, including the perception of obstetricians and family practitioners.

Unplanned, unintended, unwanted, or mistimed pregnancy can result in psychological effects on parents and possible poor outcomes in pregnancy. In the study done by Abdulwahab et al. ${ }^{20}$, the prevalence of unplanned or ambivalent pregnancy in Saudi Arabia was $46.9 \%$. In our study, the question about planning pregnancy was not part of the study. However, the data were collected about this issue. We found that around $40 \%$ of the pregnancies were unplanned, which is considered an urgent call to increase public awareness regarding the risk of unplanned pregnancy and associated outcomes. Pregnant women with epilepsy should be familiar with the im- portance of contraception to prevent this problem. In addition, all female patients with epilepsy with childbearing potential should routinely use folic acid on a daily basis. This approach is considered the safest approach to tackle this issue.

Exposure to antiepileptic drugs might affect fetal growth with a subsequently reduced head circumference in newborn babies and increased risk for small-for-gestational age. ${ }^{21}$ The evidence is limited, mainly originating from hospital-based cohorts and population-based registry studies. The clinical significance of these findings is uncertain, and the microcephaly incidence rate is not increased in newborn babies exposed to antiepileptic drugs in-utero. ${ }^{22}$ In our study, head circumference was in the normal percentile in all of the newborns, and only 12 babies (2\%) had low birth weight (below $2.5 \mathrm{~kg}$ ). This is much lower than low birth weight in developing countries $(16 \%)$ or developed countries (7\%). ${ }^{21}$ No explanation could be found for such a lower incidence in our study population.

Estimating the rate of pregnancy loss and miscarriages in pregnant women with epilepsy is difficult since many cases occur early in pregnancy and hence can pass unrecognized. ${ }^{23}$ In a study conducted in India, ${ }^{24}$ the rate of spontaneous abortion was double in pregnant women with epilepsy as compared to the general population. In another single-center study, ${ }^{25}$ spontaneous abortions occurred in 10\% of women. In our study, the results revealed a percentage of $15.6 \%$ pregnancy loss from the total number of pregnancies. This issue should be investigated more by conducting systematic nationwide research.

Based on the results of our study, we think that both monotherapy and polytherapy can be prescribed under proper monitoring. However, monotherapy causes less harm to infants in comparison with polytherapy. Whenever there is a planned pregnancy, the lowest dose achieving control of preferably a new generation antiepileptic drug should be selected for the women. Lack of research in Saudi Arabia in this subject is considered a major obstacle, and more studies need to be done with specific attention to epidemiological data and statistics. As long as a patient is following up regularly and taking the medications on time as well as her seizures are well-controlled, her neurologist should try to reduce the dose until the lowest effective dosage is reached.

Although there are several limitations regarding this study, the findings were striking, including the number of unplanned pregnancies, the number of miscarriages/abortions, and lack of antenatal/postnatal precautions and adherence to guidelines. The healthcare system in Saudi Arabia is very advanced, which is provided for free to 
the citizens. This indicates the huge demand for general public health education and the necessity of primary healthcare effective programs. The results of this small study are an urgent call for the establishment of congenital malformations registry in Saudi Arabia. In addition, specialized epilepsy clinics utilizing multidisciplinary care are highly recommended. A specific group of interest for such clinics are married women who have epilepsy and are using antiepileptic drugs since planning of pregnancy is not part of the culture in Saudi Arabia.

\section{Conflicts of Interest}

The authors declare that they have no conflicts of interest.

\section{References}

1. Karasin $\mathrm{B}$, Karasin M. Epilepsy: clinical review and surgical options. AORN J 2017; 106:393-414

2. Khuda I, Aljaafari D. Epilepsy in pregnancy. A comprehensive literature review and suggestions for saudi practitioners. Neurosciences (Riyadh) 2018;23:185-93.

3. Bech BH, Kjaersgaard Ml, Pedersen HS, et al. Use of antiepileptic drugs during pregnancy and risk of spontaneous abortion and stillbirth: population based cohort study. BMJ 2014;349:95159.

4. Viinikainen $K$, Heinonen S, Eriksson $K$, Kälviäinen R. Fertility in women with active epilepsy. Neurology 2007;69:2107-8.

5. Harden CL, Hopp J, Ting TY, et al. Practice parameter update: management issues for women with epilepsy--focus on pregnancy (an evidence-based review): obstetrical complications and change in seizure frequency: report of the Quality Standards Subcommittee and Therapeutics and Technology Assessment Subcommittee of the American Academy of Neurology and American Epilepsy Society. Neurology 2009; 73:126-32.

6. Pennell PB. Pregnancy, epilepsy, and women's issues. Continuum (Minneap Minn) 2013;19(3 Epilepsy):697-714.

7. Voinescu PE, Pennell PB. Management of epilepsy during pregnancy. Expert Rev Neurother 2015;15:1171-87.

8. Patel SI, Pennell PB. Management of epilepsy during pregnancy: an update. Ther Adv Neurol Disord 2016;9:118-29.

9. Harden CL. Pregnancy and epilepsy. Continuum (Minneap Minn) 2014; 20(1 Neurology of Pregnancy):60-79.

10. Al Bunyan M, Abo-Talib Z. Outcome of pregnancies in epileptic women: a study in Saudi Arabia. Seizure 1999;8:26-9.

11. Pennell PB. Antiepileptic drugs during pregnancy: what is known and which AEDs seem to be safest? Epilepsia 2008;49 Suppl 9:43-55.
12. Viale L, Allotey J, Cheong-see F, et al. Epilepsy in pregnancy and reproductive outcomes: a systematic review and meta-analysis. Lancet 2015;386:1845-52.

13. Tomson T, Battino D, Perucca E. Teratogenicity of antiepileptic drugs. Curr Opin Neurol 2019;32:246-52.

14. Tomson T, Landmark CJ, Battino D. Antiepileptic drug treatment in pregnancy: changes in drug disposition and their clinical implications. Epilepsia 2013;54:405-14.

15. Kaplan YC, Nulman I, Koren G. Dose-dependent risk of malformations with antiepileptic drugs: an analysis of data from the EURAP epilepsy and pregnancy registry. Ther Drug Monit 2015;37:557-8.

16. Vajda FJ, O'Brien TJ, Graham J, Lander CM, Eadie MJ. Associations between particular types of fetal malformation and antiepileptic drug exposure in utero. Acta Neurol Scand 2013;128:228-34.

17. Harden $\mathrm{CL}$, Meador $\mathrm{KJ}$, Pennell $\mathrm{PB}$, et al. Practice parameter update: management issues for women with epilepsy--focus on pregnancy (an evidence-based review): teratogenesis and perinatal outcomes: report of the Quality Standards Subcommittee and Therapeutics and Technology Assessment Subcommittee of the American Academy of Neurology and American Epilepsy Society. Neurology 2009;73:133-41.

18. Thangaratinam $S$, Fong $F, M c C o r r y ~ D$, et al. Epilepsy in Pregnancy. London: Royal College of Obstetricians and Gynaecologists, 2016;1-33.

19. Harden $C L$, Pennell PB, Koppel BS, et al. Practice parameter update: management issues for women with epilepsy--focus on pregnancy (an evidence-based review): vitamin K, folic acid, blood levels, and breastfeeding: report of the Quality Standards Subcommittee and Therapeutics and Technology Assessment Subcommittee of the American Academy of Neurology and American Epilepsy Society. Neurology 2009;73:142-9.

20. Abdulwahab A, Almotairi A, Alkhamis W, Almutiari A. Prevalence of unplanned pregnancy and its psychological effect among pregnant patients in King Khalid University Hospitals. Egypt J Hosp Med 2018;70:943-47.

21. Artama M, Gissler M, Malm H, Ritvanen A; Drug and Pregnancy Group. Effects of maternal epilepsy and antiepileptic drug use during pregnancy on perinatal health in offspring: nationwide, retrospective cohort study in Finland. Drug Saf 2013;36:359-69.

22. Laganà $A S$, Triolo $O$, D'Amico $V$, et al. Management of women with epilepsy: from preconception to post-partum. Arch Gynecol Obstet 2016; 293:493-503.

23. Tomson T, Battino D. Teratogenic effects of antiepileptic drugs. Lancet Neurol 2012;11:803-13.

24. Thomas SV, Sindhu K, Ajaykumar B, Sulekha devi PB, Sujamol J. Maternal and obstetric outcome of women with epilepsy. Seizure 2009;18:163-6.

25. Pittschieler S, Brezinka C, Jahn B, et al. Spontaneous abortion and the prophylactic effect of folic acid supplementation in epileptic women undergoing antiepileptic therapy. J Neuro/ 2008;255:1926-31. 